\title{
Analytical biophotonics
}

\author{
Jürgen Popp • Reiner Salzer
}

Published online: 13 May 2011

(C) Springer-Verlag 2011

There is a multitude of different definitions of biophotonics. Although, in many cases, biophotonics is reduced to the application of optical methods to solve problems in the life sciences, we see it, instead, in a much broader sense as a multidisciplinary research area which includes all light-based methods and technology applied not only in the life sciences but also in medicine and even to analytical problems with regard to food, environment, and security. It can be deduced from its fields of application that biophotonics is a discipline which has significant societal importance and enormous potential to meet present and future challenges in these fields.

Starting from the beginning of biophotonics, which we see as the invention of the light microscope back in the 17 th century, biophotonics has developed into a field with a multitude of subdisciplines. With regard to its connection to

Published in the special issue Biophotonics with Guest Editors Jürgen Popp and Reiner Salzer.

\footnotetext{
J. Popp $(\bowtie)$

Institut für Physikalische Chemie,

Friedrich-Schiller-Universitaet Jena,

Helmholtzweg 4,

07743 Jena, Germany

e-mail: juergen.popp@ipht-jena.de

\section{J. Popp}

Institut für Photonische Technologien e.V,

A.- Einstein-Str. 9,

07745 Jena, Germany

\section{R. Salzer}

Fachrichtung Chemie und Lebensmittelchemie,

Technische Universität Dresden,

01062 Dresden, Germany

e-mail: reiner.salzer@tu-dresden.de
}

analysis, biophotonics can be subdivided according to the dimensions of the sample of interest. Starting from molecules these include single cells, tissue, and, finally, organs and the body as a whole.

Molecular diagnostics is in the focus of point-of-care devices, which mostly contain chip-based sensors. These devices are developed to measure the amount of specific analytes, for example sugar, in blood and require, apart from a detection unit, sophisticated sample preparation also. This preparation step is often realized by microfluidics, potentially in combination with the emerging optical nanotrapping methods. Apart from that, the technology can also be used to decipher processes in cells to gain a deeper understanding of, for example, cell communication processes or the processes involved in the transformation of exogenous substances. In the latter process thiopurine methyltransferase plays a role; the activity of this was investigated by März et al. by surface-enhanced Raman spectroscopy using a lab-on-a-chip device. Also by means of Raman spectroscopy, and at the molecular level, Walter et al. investigated the reaction of bacteria to antibiotics, to detect resistance. Similarly at the molecular level the gender of fertilized unincubated chicken eggs can be determined by infrared spectroscopy imaging, as shown in the contribution by Steiner et al. As an application in environmental biophotonics, the contribution of Leidner et al. provides insight into the development of a grating coupler system which can be used to study concentration gradients in aqueous solutions.

At the level of single-cell diagnostics a prominent example of the application of photonic methods is the identification of single bacteria which can avoid the time-consuming procedure of cultivating them. Another potential field of application at this 
level, reported in the contribution of Jiang et al, is the use of tagged gold nanoparticles to enable, by means of surfaceenhanced Raman spectroscopy, the imaging of cells, in particular cancer cells, because of their high uptake.

Focussing at the tissue level, application of biophotonics methods are often centred on the detection of cancer or precancerous tissue. Cicchi et al. used fluorescence measurements to discriminate between healthy and abnormal tissue. Because steady-state fluorescence measurements usually tend to give only morphological information, they used two-photon fluorescence lifetime measurements to include also functional information and showed that this label-free method has great potential for the purpose at hand. A more mature method, by comparison, which has already found its way into clinical practice, is optical coherence tomography. Routinely used for examination of eye diseases, more and more new fields of application are being discovered, for example cardiology, dermatology, and gastroenterology, to name just a few. The review of Marschall et al. gives insight into the current state-of-theart of optical coherence tomography in these fields and details and discusses new developments and trends. Another review, authored by Walther et al., concentrates on the application of modern OCT techniques in ophthalmology, angiology, and emergency medicine and/or intensive care. Also label-free, but with the advantages of also providing molecular information apart from morphology and at higher resolution, Raman-based spectroscopic methods have their special merits. Beleites et al. used Raman spectroscopy to classify astrocytomas, which are highly malignant brain tumours. This can be seen as a step towards a new diagnostic tool to support surgical treatment of such tumours. The actual state of development of this kind of intra-operative optical diagnosis by vibrational spectroscopy is reviewed in the contribution by Stelling et al. Diagnosis at the level between tissue and organ is the subject of the work of Benalcazar et al., who used nonlinear interferometric vibrational imaging, a technique based on coherent anti-Stokes Raman scattering, to image skin. Again, this technique has potential to be used in optical biopsies and to support the pathologists of the future.

As guest editors, it is our special pleasure to present this collection of papers and reviews. Of course it is impossible to cover the whole field of analytical biophotonics within this special issue, but we hope it provides some new insight into this interesting and expanding field. We thank all of the authors for their contributions and, especially, the editorial staff of $\mathrm{ABC}$ for their valuable help in preparing this special issue.

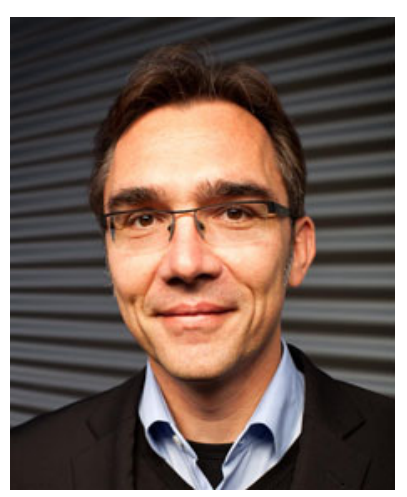

Jürgen Popp Is a full professor at the Friedrich-Schiller University of Jena, Germany, where he holds a chair of physical chemistry. In June 2006 he also became the Scientific Director at the Institute of Photonic Technology. His work has been awarded the Faculty Prize of Chemistry (1995) by the "Bayerischer Habilitationsförderpreis" (1997), the "Förderpreis der Würzburger Korporationen" (2001), and the Kirchhoff-Bunsen Award (2002).

J. Popp's research interests are mainly centred around the development and application of frequencyresolved, time-resolved, and spatially resolved innovative laser spectroscopic methods and techniques ranging from the UV into the NIR region for the derivation of structure-activity or dynamic relationships. This type of investigative approach is essential in resolving important questions in fields of biology, medicine, pharmacy, and astronomy, and in the environmental and material sciences. In particular his expertise in the field of Raman spectroscopy and in the development of innovative Raman techniques should be emphasized. The results obtained by J. Popp have been published in more than 200 scientific articles in premier peerreviewed journals. He is inventor named on eight patents in the field of spectroscopic instrumentation. Since 2008 he has been coordinator of the Network of Excellence for Biophotonics- -Photonics4Life".

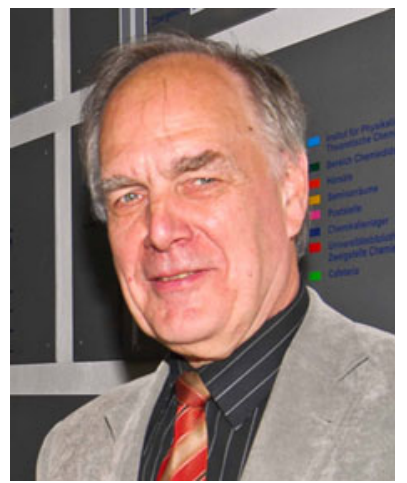

Reiner Salzer Retired as Professor of Analytical Chemistry at the Technische Universität Dresden, Germany, in 2007. He obtained his academic degrees from the University of Leipzig, before taking up his appointment at the TU Dresden. His main scientific interests include molecular monitoring for early diagnosis of diseases, integration of biologically active functions into polymers, and electronic media in university education. Professor Salzer has authored 14 patents in different fields of analytical chemistry, and over 250 books and scientific publications.

Professor Salzer has been elected on to a variety of national and international boards. He is a member of the Norwegian Academy of Science, a recipient of the Emich Plaque of the Austrian Society of Analytical Chemistry (2007) and of the Clemens Winkler Medal of the Division Analytical Chemistry of the German Chemical Society (2011). Professor Salzer is a long-time advisory board member for analytical and spectroscopic journals. He served as President of the Division Analytical Chemistry of the German Chemical Society, and is National Delegate to the Division of Analytical Chemistry of EuCheMS, where he is Head of the Study Group Education. He was elected as Vice-Chairman of the ECTNA Label Committee for the Chemistry Eurobachelor and Chemistry Euromaster and is currently involved in the development of a quality scheme for a Eurodoctorate label. 